\title{
OBSERVATIONS
}

\section{ON \\ THE CATARACT.}

By BENJAMIN TRAVERS, Ese.

DEMONSTRATOR OF ANATOMY AT GUY'S HOSPITAL;

SURGEON TO THE HON. EAST.INDIA COMPANY, AND TO THE

LONDON INFIRMARY FOR DISEASES OF THE EYE.

Read July 13, 1813.

OPACITY is the result of inflammation in a transparent part, as is seen in the scarf-skin, the conjunctiva, the cornea, the crystalline and vitreous humours of the eye, and their membranes. If the inflammation is arrested before it reaches the stage of effusion, the opacity, which probably depends upon the turgescence of the colourless vessels, is partial; and the part recovers its transparency when the inflammatory action ceases. This is proved by the haziness of the cornea in acute inflammation of the conjunctiva, accompanied by dimness of vision; a symptom which gradually goes off after the inflammation is subdued. When effusion has taken place even in a moderate degree, as in the nebula of the cornea, the opacity is more slowly removed, and seldom perfectly, even long after the inflammation has ceased. Where a deposition has taken place in consequence of a breach 
of the natural texture, as after an ulcer of the cornea, the opacity, greater or lés according to the extent of the deposition; is permanent. Inflammation is the only cause of cataract open to obser-. vation. The simplest example of it is afforded by the accidental wound of the crystalline by a penetrating instrument; in this case the opacity is partial, and the cicatrix of the wound is the centre of the opaque spot. Another frequent example is the cataract following acute inflammation of the choroid and iris, whether arising spontaneously or from injury, as a violent contusion of the eye. In this case the opacity is sometimes diffused over the capsule of the lens which adheres to the contracted pupil; sometimes it is only a central spot, and the iris seems to adhere to a transparent part of the capsule.

A conformation of body favouring a determination of blood to the brain, or frequent exposure of the eye to the stimuli of heat and light in more than ordinary intensity, or the habitual vision of minute objects, in a depending position of the head, by which an undue proportion of blood is thrown upon the organ, commonly induce opacity of the crystalline or of the retina; which in one species of amaurosis turns of a green yellow colour, and becomes distinctly visible.

Cataracts are very frequently of spontaneous occurrence in persons of advanced years, in whom no signs of inflammation have preceded the complaint. 
Transparent parts obviously tend to become opaque in age, as may be instanced by the want of clearness of complexion in old persons, and the arcus senilis, as it is called, which is an opacity without inflammation, encroaching upon the cornea. The very minute serous vessels of the crystalline run in the cellular substance which unites the lamellæ. This interstitial texture is probably absorbed in age, and the vessels may be gradually obliterated by compression *; but this must be matter of conjecture.

Cataracts are also formed in utero, and I have rarely observed in the subjects of congenital cataract other marks of deranged or defective organization. Some other and more subtle cause of opacity must therefore be admitted.

The cataracts of new-born children and of aged persons exhibit very opposite appearances. In congenital cases the opacity most frequently appears in the central nucleus, the interior denser structure demonstrated in the healthy lens by Petit, and is either stationary, or enlarges equally

* A change in the action of so minute and remote a system of vessels we should not expect to discover otherwise than by its local effects. Changes in other organs, similar to those which produce the different kinds of cataract, are familiar to our ordinary experience. Thus we see the matter of secretions altered, loose interstitial texture consolidated by excess of deposition, or obliterated by absorption; changes, which, in lymphatic glands, and parts framed for less nice and delicate purposes than the organ of vision, are less obvious, although equally subversive of their functions. 
and slowly in a circle. The nucleus is sometimes not bigger than a pin's head in the centre of the transparent lens; but more commonly it is of the size of the contracted pupil, so that the child habitually knits his brow's, or screens his eyes with his hand, to obtain that state of the pupil which he finds necessary to his vision. The fluid and capsular cataracts are exceptions to this observation. It is well known that adult subjects of cataract see better in moderate than in strong light, but in a much less degree; for the opacity is in them more diffused, so as very faintly, if at all, to exhibit a nucleus; and a dilatation beyond a natural one, I mean that obtained by the belladonna, though it enlarges somewhat the field of light, seldom permits of vision. The opacity commonly appears of equal consistency from the origin of the complaint, and in its progress the light is shut out from the whole sphere of the pupil. The hard cataract affords a partial exception to this remark, in which the nucleus, though imperfectly defined, is generally to be distinguished.

The opacity is sometimes simply capsular, which is known by the uniform nebulous tenuity of the opaque membrane stretched over the transparent lens, and rendered more distinct by the dark tint reflected from the choroides. The cataract appears to be prominent in the pupil, which is sometimes slightly irregular. In this case, which is considered to be an incipient state of 
the cataract, as by the consequent opacity or absorption of the lens it becomes more dense and distinct, the quantity of light admitted is considerable.

More frequently the opacity is simply lenticular, which is known by the cataract appearing more dense, voluminous, and varied in its colour and texture, and, in relation to the plane of the iris, deeper seated, by the circularity of the pupil, and the greater degree of blindness in the natural state of dilatation. The motions of the pupil being regulated by the quantity of light which is admitted to the retina, its size depends upon the texture and bulk of the opaque lens, $i$. e. a very dense cataract keeps it dilated by excluding light from the retina ; a very bulky one by mechanically distending it. In most cases of congenital cataract, and in some of mature age, the dilatation by belladonna discovers a defined margin to the opacity, and a transparent circle beyond it, and therefore adds considerably to the patient's perception of light.' I have known patients in this state, who were of an age to judge for themselves, decline the operation, content with the vision they enjoyed by the use of the belladonna. In such cases, however, a tolerable vision has been previously enjoyed, owing to the smallness of the opaque nucleus compared with the transparent portion of the lens. And in all cases the vision of near objects is confused, if not totally bedimmed, by the enlargement of the pupil with the belladonna, although that of distant ones is 
clear and distinct. Where a transparent circumference has been discovered after dilating the pupil by the belladonna, I have never seen the capsule opaque, and I believe this black rim may be considered as diagnostic of the transparency of the capsule. Where the lenticular opacity is diffused, this sign of a transparent capsule is of course wanting.

The opacity is sometimes much deeper seated, so that you look at it through the transparent capsule and lens. It is here generally circumscribed, but irregularly shaped; and often, from its tenuity and depth of situation, escapes the observation even of oculists. This is usually considered to be a third seat of opacity, distinct from the former, viz. in the posterior covering of the lens. I do not find, upon repeated and strict examination, any proper capsule investing the lens, $i$. $e$. which admits of being removed with it. It may be necessary to a right understanding of this structure, briefly to describe it. The tunic of the vitreous humour advances to the ciliary body, there it separates into two laminæ, which, when contiguous to the margin of the crystalline, adhere closely to each other, forming the sacculated circle (canal godronné) described by Petit, which is capable of being inflated around the margin of the lens. This canal corresponds in breadth to the breadth of the ciliary processes, and is marked by them anteriorly. The anterior lamina, which is the more dense of the two, covers the crystalline in front; 
the posterior lines the fossula of the vitreous humour. There is no communication betwixt the canal of Petit, the vitreous humour, and the crystalline capsule. They are all distinct from each other, and must be inflated distinctly, if perfect. The crystalline, it will appear from this description, is incased in a duplicature of the vitreous capsule. The different texture of these laminæ adapted to their respective uses, (the one properly belonging to the crystalline, and supporting the whole lens in its place; the other proper to the vitreous, and covering a very small portion of the humour, which is sufficiently supported by the crystalline itself) and likewise the close investiture of the margin of the lens, which interrupts continuity, for it prevents the passage of air, explain why they are so seldom similarly affected in disease. The posterior opacity before described is therefore seated in the proper tunic of the vitreous humour. Thus much on the situations of the opacity forming cataract.

The varieties of consistency, colour, and figure, are numerous. With regard to consistency, we have the fluid or milky, the flocculent or fleecy, the caseous or doughy, and the compact or hard cataract. The fluid lens is, I believe, rarely contained in a transparent capsule. The latter, in my experience, has been partially opaque, presenting a dotted or mottled surface. The capsule appears in contiguity with the margin of the pupil, 
and as if projecting in it, and the opaque spots upon it are most distinguishable when it is viewed laterally. The second usually resembles, in appearance, flakes of snow irregularly heaped, being visibly of a loose and broken texture, and the larger masses intersected by semi-transparent lines: the arrangement is sometimes regular and uniform, being either foliated or radiated. The capsule is sometimes semi-opaque, but more frequently transparent. The third is the cataract of greatest bulk, impeding the motions of the pupil, having a heavy and dense appearance, uniformly opaque, a clouded not a fleecy whiteness, and sometimes a greenish or dirty white tinge. The fourth appears deep seated, of a brown yellow, or amber colour, most dense in the centre; if entirely opaque, flat upon the surface, over which the iris plays freely. The second and third species are most commonly met with; the first and fourth are comparatively rare.

The description of such appearances is difficult, and might appear over-minute; but to an eye much accustomed they admit of ready distinction, and the distinction is of the highest importance, as the operation should be selected accordingly. To the first and second species, formerly regarded as incurable on account of their softness, the operation performed by the late Mr. Saunders is admirably adapted. To the two latter, the operation of couching or extraction is best suited. 
In the description given the capsular opacity is not included; for when the capsule is completely opaque, we can hardly judge of the texture of the lens. But where the complete opacity of the capsule exists, the lens is commonly diminished in bulk; it undergoes a waste after the opacity of the capsule, so as in process of time to become a membranous cataract. This I conceive to be owing to the obliteration of the vessels of the capsule, from which those of the lens are derived. When the capsular opacity is congenital, it is either purely capsular, or only a very small piece of lens remains. When the capsule turns opaque from injury, the lens is soon greatly reduced in bulk, as appears from the falling in or concavity of the iris which loses its support, and is demonstrated in the operation. This observation renders the operation with the needle appropriate to the cataract in which the capsule is opaque, in cases which are not very recent. When the capsule of the vitreous humour is the seat of the opacity, I have not ob. served that the lens undergoes any diminution, nor have I yet ascertained the remediableness of this case*. The membranous or purely capsular cataract is a form of the disease which appears to me to require a somewhat different operation.

The fluid cataract commonly requires only the

- I avail myself of the opportunity of a second edition, to say that I have since seen this form of the disease removed by an pperation.

VOL. IV. 
central aperture of the capsule; it flows out into the anterior chamber, and mixes with the aqueous humour, which, by the absorption of the opaque particles, in a few days recovers its transparency. It is to be observed, however, that this form of cataract requires to be treated with caution. I have seen two instances in which the simple discharge of the fluid was followed by severe inflammation, by which the process of absorption was arrested, as appeared from the permanently turbid state of the humour. This does not happen where the whole substance of the lens is changed. The milk-like fluid in these cases concealed a solid bed of lens, which, by the free laceration of the capsule, was set at liberty, and oppressed the iris.

The flocculent cataract readily undergoes solution in the aqueous humour, when the capsule is freely opened, and its texture broken down by the needle:*

* The attempt to procure a solution of the opaque crystalline in the aqueous humour, was suggested by an accident, in which the crystalline being wounded, became opaque, and was removed by this process. I bave seen many examples of the same fact. Among the miracles recorded to have been wrought at the tomb of the Abbé Paris, is the gradual restoration of sight to a young man who became blind after a puncture of the eye with an awl, which caused the discharge of the aqueous humour. (See Paley's Evidences of Christianity, vol. I. p. 380.) This miracle admits of a more satisfactory explanation than has yet been offered, as the familiar case of a cataract formed by the wound, and undergoing absorption. 
While any portion of the capsule preserves its connection, it continues to be nourished, and of course resists absorption; but an insulated portion of capsule is dissolved and absorbed, like any other matter extraneous to the circulation. These facts are ascertained by the aperture in the capsule remaining ever after unchanged in dimension, and if it be equal to the natural aperture of the iris, so that its broken margin cannot be seen in the pupil, it will always appear upon dilating the pupil with the belladonna. If the aperture be made of a given size and figure, as for example, the figure of a small diamond, in the centre of the capsule, it will ever after retain its figure and dimensions. But where the aperture is ultimately of the full size of the pupil, it is evident that the portion of capsule that has disappeared, must have undergone solution: and absorption with the lens. The transparent capsule is tense and contractile as well as delicate in texture, so that a wound made in it is enlarged by the retraction of its sides; but the retraction without loss of substance would be very insufficient. to account for the magnitude of the aperture, and we continually see that a mere puncture or cut of the transparent capsule, from accidents with pins and scissors, unites by adhesion, forming an opacity. In the opaque capsule, which has no contractility, it is still more decidedly proved, that the: aperture of the size of the pupil must be produced by.the laceration of the capsule in fragments; and

U 2 
the absorption of these fragments together with those of the lens.

The reasons why the operation of Mr. Saunders is objectionable in the two latter species of cataract, are, first, the degree of force required to break down these cataracts with the needle, sufficiently to undergo a quick solution in the aqueous fluid ; and, secondly, the danger of dislocating the lens in the attempt. If the operation upon a cataract of firm consistence is conducted with the caution which experience dictates, the process of solution is tediously slow, and the operation must be repeated many times; but this is the least evil. If the operator, after having opened the capsule in the centre, acts with the same freedom as upon the soft species of cataract, the unsupported lens falls forward, or revolves and turns edge foremost in the pupil, so as to put the iris on the stretch; or it passes entire into the anterior chamber. : If he succeed in dividing it, large and solid masses will press upon the iris, wedge in the pupil, or pass into the anterior chamber, and the consequences be the same in kind though less in degree. Even where the operation is confined to the aperture of the capsule, and the lens has been scarcely touched, I have known the eye destroyed by the accidental falling of the lens through the aperture and pupil into the anterior chamber; a slow inflammation of the iris ensues, which not only produces the most distressing symptoms, but 
admits of little, if any, alleviation, until the mechanical pressure is taken off. This event, so much to be desired, is unfortunately protracted, for the process of solution is arrested by an adventitious coating of lymph, which is effused by the inflamed vessels, and invests the extraneous body. This lymph becomes partially organized, and the case terminates in obliteration of the pupil and anterior chamber, or by the sympathy of the retina with the suffering iris and choroid, in incurable amaurosis. Having more than once observed the protrusion of the lens, and the consequent inflammation of the iris to ensue, where I had studiously endeavoured to prevent it, I was led to suppose. this might be owing to the previous dilatation of the pupil by belladonna, by which the lens was deprived of support. I therefore omitted the previous use of the belladonna where the cataract was substantial and firm. It was then applied to the eyebrow an hour or two after the operation, with a view of preventing the adhesion of the pupil to the capsule of the lens; but under the influence of the bella. donna, I repeatedly observed, that the unsupported lens came forward, and the symptoms of pressure commenced. I therefore deferred its employment until a time sufficient had elapsed for the renewal of the aqueous humour, which, I conceived, might resist the pressure of the lens. By this precaution the accident was effectually prevented, no symptom of inflammation followed, and the advantage of keeping the pupil circular was equally obtained. 
It may be right to observe here that when the belladonna is used with this view, the interval of its application should be sufficient to admit of the recovery of the pupil, or it will be permanently dilated. With every precaution, however, the operation with the needle upon the full and firm cataract, is either distressingly tedious, or what is more to be objected, destructive to the organ, and very trying to the health and spirits of the patient. In such cases $I$ have, therefore, relinquished the operation, which was very ingeniously conceived, and for its simplicity appeared to me to deserve a full and fair trial of its merits. It is due to the projector of the operation to state, that its superiority was distinctly asserted by him only in the cases of soft and capsular cataract, as may be seen by a reference to his essay on that subject, in his posthumous work edited by Dr. Farre. At page 173 of the volume referred to, the editor observes, "In the adult, if the texture of the lens is nearly uniform and permeable, the cure is completed in a space of from three to five months; but if the texture is firmer and the nucleus large, the cure cannot be accomplished in less than seven months. On this account the author, who thought highly of extraction, and performed this operation with dexterity and success, was inclined to extract the lens when its texture was unusually hard. The editor cannot assert that he would ultimately have conceded thus much in favour of extraction. It was intended, that such decision should result from 
a very long and impartial trial of both operations. With respect to the softer lens, or the capsular cataract, he was satisfied of the superiority of his operation."

In performing this operation for the soft cafaract the operator may pass his needle through the cornea or the sclerotic. The former mode commands the advantage of giving no pain, exciting but slight inflammation, and ensures the laceration of the transparent capsule, which is sometimes so elastic that the operator, whose eye is not well accustomed, is in danger of not opening it in the centre, or of wounding the interior surface of the cornea. This is especially to be feared in operating behind the iris, when the lens is, at the same time, much diminished by absorption, as in children; so that the capsule moves before the needle instead of resisting it. Few cases of lenticular cataract occur, to which this method is appropriate, and in which it is adviseable to perform the posterior operation. There is more pain, more inflammation, more danger of displacing the lens tian in the operation through the cornea. But when the capsule is opaque and the lens diminished in bulk, either spontaneously or in consequence of former operations, so as to have receded from the pripil; the posterior operation is more eligible, as the operator readily ascertains the effect of the needle upon the capsule, and directs its movements to the best advantage; while the inflammation is always moderate in proportion as the lens is sman. In. 
deed, in the purely capsular cataract of the adult, and the half absorbed cataract of children, it is so slight as scarcely to confine the patient. When a cataract of firm consistence has undergone a partial absorption from one or more operations with the needle, and still does not readily separate into fragments, the introduction of the needle, posterior to the iris, gives the surgeon the advantage of couching it. This I have often done to the great satisfaction of the patient, who escapes, owing to the diminished bulk of the lens, the inflammation which occasionally follows the primary operation of couching. It was my intention to point out the circumstances which should determine the election of couching or extraction in the two latter species of cataract, and to shew the value of these operations relatively to each other and to that by solution, as the deliberate result of an impartial investigation of all. This I shall make the subject of a future communication.

I have annexed a plate, representing the appearances of the several species of cataract described. Varieties may be referred to one or other of these as a standard. If the operation proper to the case be selected, each will maintain its credit, as neither of them is applicable to all cases. I believe, upon inquiry, it will be found that the exclusive preference given by operators to one method, and, consequently, the frequent mal-appropriation of the remedy to the case, has proved the most common cause of failure in the treatment of this disease. 

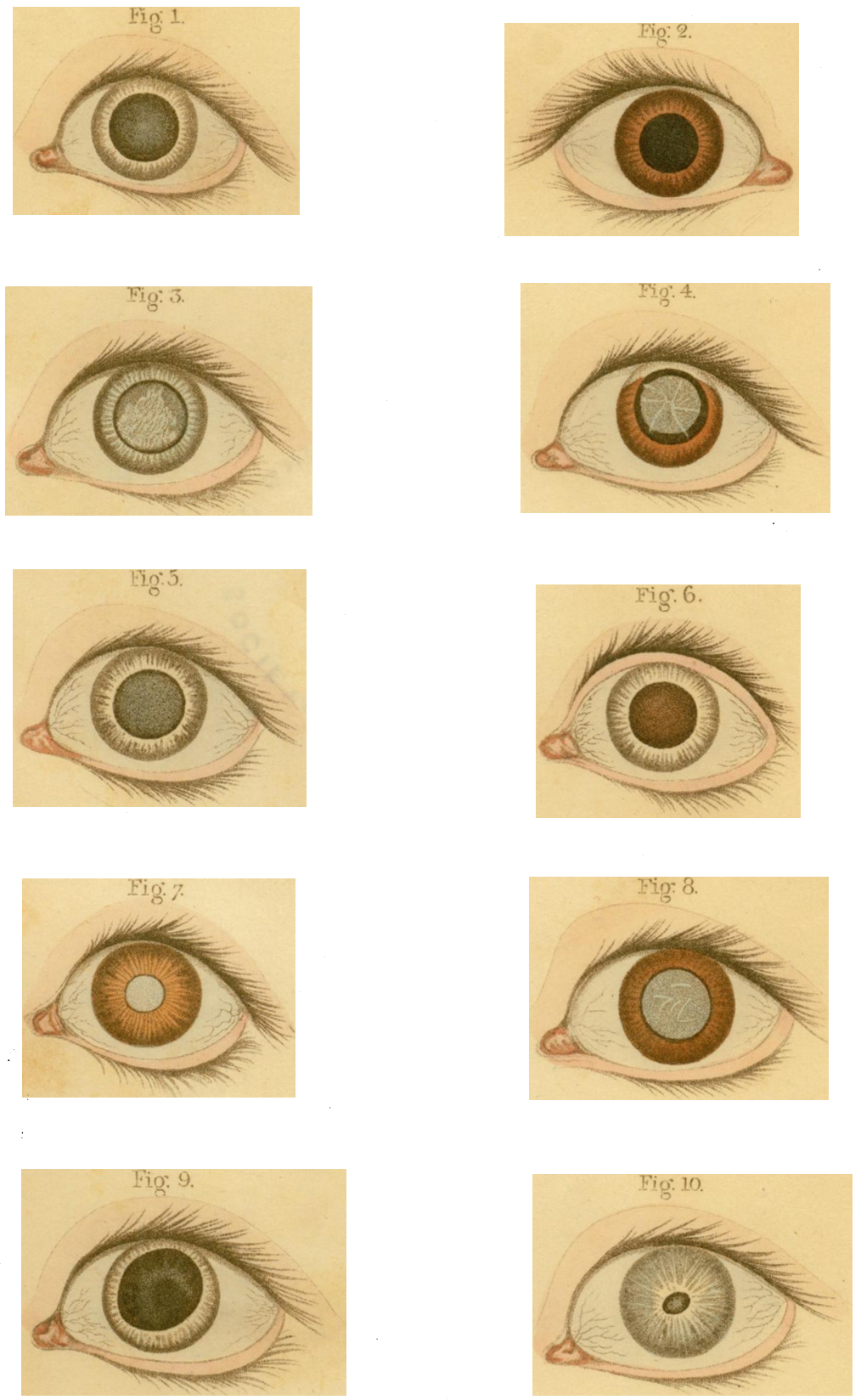
Vol. IV.
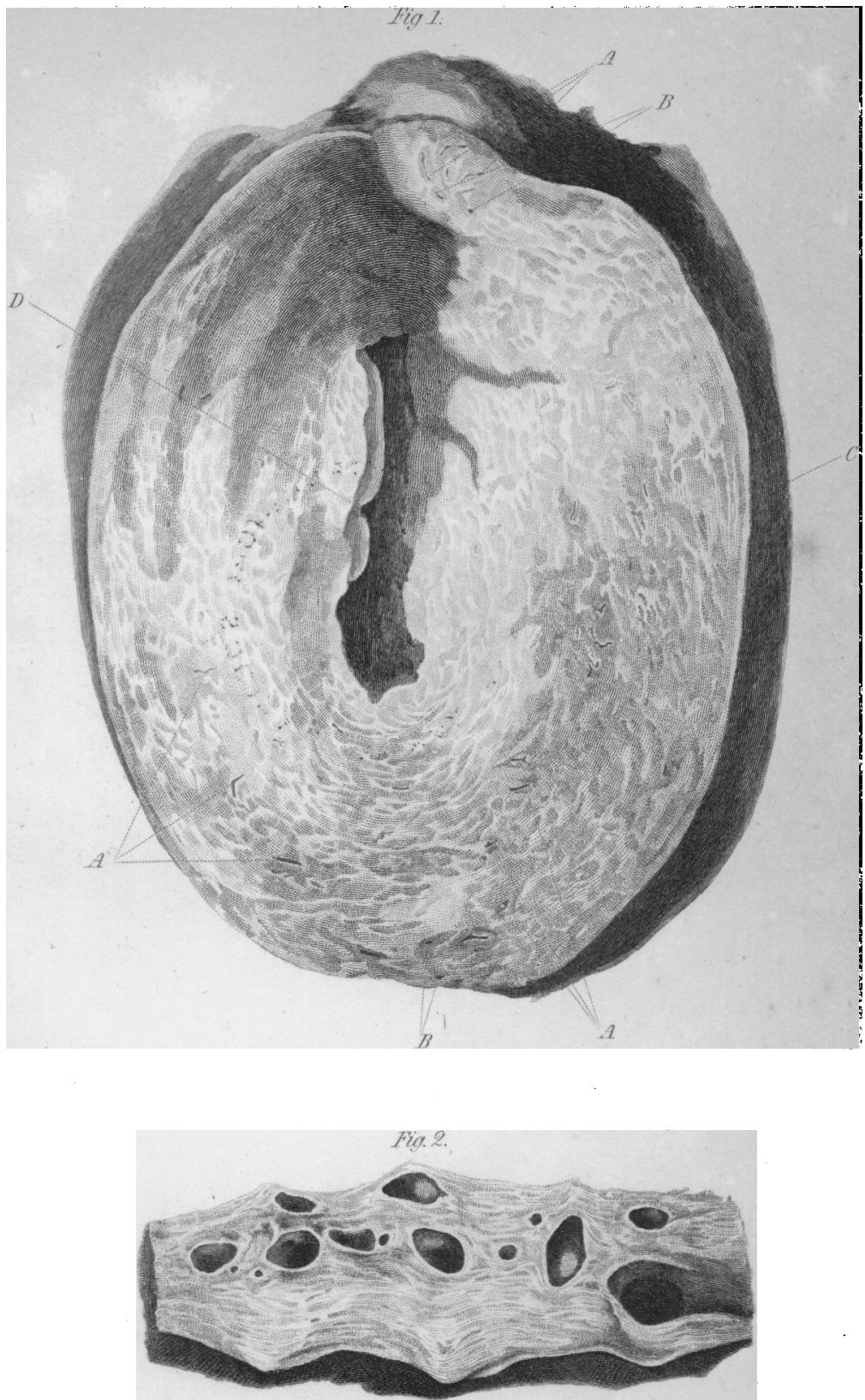